\title{
Protocolo diagnóstico de las miocardiopatías genéticas
}

\author{
Diagnostic protocol for genetic cardiomyopathies
}

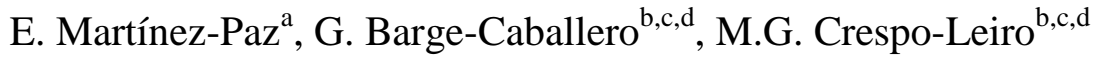 \\ ${ }^{a}$ Servicio de Cardiología. Complejo Asistencial Universitario de León. León. España \\ ${ }^{b}$ Unidad de Insuficiencia Cardíaca Avanzada y Trasplante Cardíaco. Servicio de Cardiología. Complexo \\ Hospitalario Universitario A Coruña. La Coruña. España \\ ${ }^{c}$ Centro de Investigación Biomédica en Red (CIBERCV). Instituto de Salud Carlos III. Madrid. España \\ ${ }^{d}$ Instituto de Investigación Biomédica de A Coruña (INIBIC). La Coruña. España
}

\begin{abstract}
Resumen
Características generales. Las miocardiopatías genéticas engloban un grupo heterogéneo de enfermedades. Algunas afectan de forma aislada al músculo cardíaco, en otros casos forman parte del espectro de una enfermedad sistémica. Con frecuencia comienzan en la juventud, constituyendo una causa importante de morbimortalidad en los pacientes afectados.

Diagnóstico. Existen manifestaciones clínicas, alteraciones electrocardiográficas y pruebas de imagen que permiten el diagnóstico de la afectación estructural cardíaca. Los avances en el campo de la genética han permitido el conocimiento de un amplio porcentaje de mutaciones causales de estas enfermedades. Una vez realizado el diagnóstico del paciente afectado, es necesario ampliar el estudio a la familia, para así identificar a individuos asintomáticos y realizar una estratificación pronóstica.
\end{abstract}

\begin{abstract}
General characteristics. Genetic cardiomyopathies encompass a heterogeneous group of diseases. Some affect the heart muscle in isolation, while in other cases they form part of the spectrum of a systemic disease. These diseases frequently start in youth and represent a significant cause of morbidity and mortality in those affected by the disease.

Diagnosis. There are clinical manifestations, electrocardiographic abnormalities and imaging tests that enable the diagnosis of cardiac structural involvement. Advances in the field of genetics have revealed a high rate of causal mutations of these diseases. Once the affected patient has been diagnosed, the study needs to be extended to the family to thereby identify asymptomatic individuals and to perform a prognostic stratification.
\end{abstract}

\section{Palabras clave}

Miocardiopatía; Genética; Familiar

Keywords

Cardiomyopathy; Genetics; Familial 


\section{Introducción}

Las miocardiopatías son enfermedades en las que el músculocardíaco es estructural y/o funcionalmente anormal, en ausencia de hipertensión arterial, enfermedad coronaria, valvular o cardiopatía congénita suficiente para causar la afectación miocárdica observada ${ }^{1}$. La primera clasificación se estableció en 1980 y categorizaba las miocardiopatías en cuatro tipos: miocardiopatía hipertrófica $(\mathrm{MCH})$, miocardiopatía dilatada (MCD), miocardiopatía restrictiva y fenotipo indeterminado ${ }^{2}$.

En el año 2008, el Grupo de Trabajo de las Enfermedades del Miocardio y Pericardio de la Sociedad Europea de Cardiología definió una nueva clasificación en la que reconocía la existencia de una quinta entidad, la miocardiopatía arritmogénica. Cada una de ellas se subdivide a su vez en dos formas: genética/familiar o no genética/no familiar ${ }^{1}$ (tabla 1). Por último, más recientemente se ha descrito el sistema nosológico MOGE(S), que incorpora además del fenotipo morfofuncional (M: morphofunctional phenotype), una descripción de los órganos afectados aparte del corazón $(\mathrm{O}$ : organ(s) involvement), el patrón de herencia (G: genetic inheritance pattern), la connotación etiológica o enfermedad/sustrato subyacente (E: etiologic annotation) y la clase funcional (S: functional status of the disease $)^{3}$.

Tabla 1. Ejemplo de diferentes mutaciones y enfermedades sistémicas que causan miocardiopatías

\begin{tabular}{|c|c|c|c|c|}
\hline $\mathrm{MCH}$ & MCD & MCA & MCR & Fenotipo indeterminado \\
\hline $\begin{array}{l}\text { Genético/familiar } \\
\text { Mutaciones proteínas sarcoméricas: cadena } \\
\text { pesada de la } \beta \text {-miosina (MYH7), proteína C } \\
\text { de unión a la miosina cardíaca (MYBPC3), } \\
\text { troponinas cardíacas: T (TNNT), I (TNNI), C } \\
\text { (TNNC), titina (TTN), cadena ligera esencial } \\
\text { de la miosina (MYL3), etc. } \\
\text { Otras mutaciones genéticas: } \\
\text { Enfermedades neuromusculares: ataxia de } \\
\text { Friedrich } \\
\text { Enfermedades metabólicas: enfermedad de } \\
\text { Danon, enfermedad de Fabry, quinasa de la } \\
\text { AMP (PRKAG2), enfermedades } \\
\text { mitocondriales } \\
\text { Síndromes malformativos: Noonan, Leopard } \\
\text { Amiloidosis familiar ATTR }\end{array}$ & $\begin{array}{l}\text { Familiar, gen } \\
\text { desconocido } \\
\text { Mutaciones proteínas del } \\
\text { citoesqueleto: distrofina, } \\
\text { desmina } \\
\text { Mutaciones proteínas de } \\
\text { membrana: lamina A/C, } \\
\text { emerina } \\
\text { Mutaciones proteínasc } \\
\text { sarcoméricas: MYH7, } \\
\text { MYBPC3, troponinas, } \\
\text { actina } \\
\text { Mutaciones genes } \\
\text { mitocondriales } \\
\text { Mutaciones proteínas } \\
\text { desmosómicas: } \\
\text { desmocolina, } \\
\text { desmoplaquina, } \\
\text { desmoglobina, } \\
\text { placoglobina, placofilina }\end{array}$ & $\begin{array}{l}\text { Familiar, gen } \\
\text { desconocido } \\
\text { Mutaciones de } \\
\text { proteínas } \\
\text { desmosómicas: } \\
\text { placoglobina, } \\
\text { desmogleína, } \\
\text { desmoplaquina, } \\
\text { desmocolina } \\
\text { Receptor cardíaco } \\
\text { de rianodina } \\
\text { (RyR2) } \\
\text { Filamina } \\
\text { Factor de } \\
\text { crecimiento } \\
\text { transformante beta } \\
\text { (TGFb) }\end{array}$ & $\begin{array}{l}\text { Mutaciones proteínas } \\
\text { sarcoméricas: TNNI, } \\
\text { TNNI 3, MYL3 } \\
\text { Amiloidosis familiar } \\
\text { Desminopatías } \\
\text { Pseudoxantoma } \\
\text { elástico } \\
\text { Hemocromatosis } \\
\text { Enfermedad de Fabry } \\
\text { Glucogenosis }\end{array}$ & $\begin{array}{l}\text { Ventrículo izquierdo no } \\
\text { compactado } \\
\text { Síndrome de Barth } \\
\text { Lamina A/C } \\
\text { ZASP }\end{array}$ \\
\hline \multicolumn{5}{|l|}{ No genético/no familiar } \\
\hline $\begin{array}{l}\text { Hijo de madre diabética } \\
\text { Secundaria a drogas } \\
\text { Amiloidosis AL, amiloidosis senil } \\
\text { Entrenamiento deportivo }\end{array}$ & $\begin{array}{l}\text { Miocarditis: infecciosa, } \\
\text { tóxica } \\
\text { Etílica } \\
\text { Eosinofílica } \\
\text { Drogas } \\
\text { Periparto } \\
\text { Trastornos endocrinos } \\
\text { Déficit nutricional } \\
\text { (tiamina, carnitina, } \\
\text { selenio, hipofosfotemia, } \\
\text { hipocalcemia) } \\
\text { Taquimiocardiopatía }\end{array}$ & Inflamación & $\begin{array}{l}\text { Amiloidosis AL } \\
\text { Esclerodermia } \\
\text { Fibrosis } \\
\text { endomiocárdica } \\
\text { Síndrome } \\
\text { hipereosinofílico } \\
\text { Fármacos: metilsergida, } \\
\text { serotonina, } \\
\text { antraciclinas } \\
\text { Afectación cardíaca por } \\
\text { síndrome carcinoide } \\
\text { Radiación }\end{array}$ & $\begin{array}{l}\text { Miocardiopatía de estrés } \\
\text { (Tako-Tsubo) }\end{array}$ \\
\hline
\end{tabular}

MCA: miocardiopatía arritmogénica; MCD: miocardiopatía dilatada; MCH: miocardiopatía hipertrófica; MCR: miocardiopatía restrictiva. 
El subgrupo de las miocardiopatías genéticas constituye un conjunto de enfermedades que tienen en común una serie de características:

1. Presentación familiar: cuando existe una persona afectada, se debe estudiar a todos los familiares de primer grado, puesto que pueden haber heredado la enfermedad. La identificación de familiares afectados tiene impacto pronóstico, puesto que la primera manifestación clínica es en ocasiones la muerte súbita.

2. Base genética: se puede hacer un diagnóstico genético de la enfermedad. En algunas miocardiopatías se encuentran identificadas más del $50 \%$ de las mutaciones causales ${ }^{4}$.

\section{Diagnóstico}

La afectación estructural cardíaca se identifica utilizando una combinación de datos clínicos y los hallazgos de diversas pruebas complementarias (como el electrocardiograma -ECG-, el ecocardiograma transtorácio o la resonancia magnética cardíaca). Si existen manifestaciones a otros niveles (por ejemplo, neurológicas o renales), se debe descartar la presencia de enfermedades que cursen con afectación multiorgánica.

Antecedentes personales

Los antecedentes personales incluyen la presencia de manifestaciones cardíacas (síncope, palpitaciones, disnea o dolor torácico) y extracardíacas, que pueden coexistir en enfermedades sindrómicas 5 .

\section{Historia familiar}

Debe recogerse un árbol familiar completo que incluya tres generaciones: es necesario averiguar la presencia de antecedentes de muerte súbita, enfermedades musculares y familiares con diagnóstico de insuficiencia cardíaca o sometidos a trasplante cardíaco. El árbol familiar aporta pistas sobre el patrón de herencia de la enfermedad: autosómico dominante (individuos afectados en todas las generaciones), autosómico recesivo (padres no afectados, consanguíneos), herencia ligada a X (mujeres portadoras o con afectación leve y hombres con afectación severa) o herencia maternolineal (solo las mujeres transmiten la enfermedad, lo que típicamente ocurre en las enfermedades mitocondriales) ${ }^{5}$.

Síntomas y signos

A continuación se describen algunos de ellos y las enfermedades en que suelen encontrarse.

1. Retraso mental: enfermedades mitocondriales, enfermedad de Danon, síndrome de Noonan, distrofinopatías (Duchenne, Becker, MCD ligada a X).

2. Sordera neurosensorial: enfermedades mitocondriales, enfermedad de Fabry, síndrome de Leopard.

3. Afectación ocular: enfermedad de Danon (retinitis pigmentaria), enfermedad de Fabry (córnea verticilata).

4. Alteraciones dermatológias: enfermedad de Fabry (angioqueratomas, hipohidrosis), síndrome de Leopard (manchas «café con leche»), síndrome de Carvajal y enfermedad de Naxos (queratoderma palmoplantar, cabello lanugo).

5. Alteraciones en la marcha: ataxia de Friedrich.

6. Parestesias, anomalías sensoriales, dolor neuropático: enfermedad de Fabry.

7. Miotonía: distrofia miotónica tipo 1 y 2.

8. Debilidad muscular: distrofinopatías, desminopatías, distrofias miotónicas, glucogenosis. 
Estudios de laboratorio

Según la sospecha clínica ha de solicitarse alguno de los siguientes estudios.

1. Hemograma, pruebas de función renal y hepática.

2. Estudios de autoinmunidad: en el contexto de una MCD e historia familiar de enfermedad autoinmune, ha de solicitarse la determinación de autoanticuerpos anticardíacos y no organoespecíficos.

Electrocardiograma

Algunas alteraciones electrocardiográficas pueden orientar el diagnóstico (tabla 2). Sin embargo, con frecuencia el ECG es normal en fases iniciales de la enfermedad.

Tabla 2. Patrones electrocardiográficos que sugieren diagnósticos concretos, agrupados según el fenotipo cardíaco predominante

\begin{tabular}{|c|c|c|}
\hline Fenotipo & Hallazgo & Enfermedades específicas \\
\hline \multirow[t]{6}{*}{$\mathrm{MCH}$} & Ondas Q en cara inferior y lateral & $\mathrm{MCH}$ \\
\hline & $\begin{array}{l}\text { Criterios de hipertrofia ventricular izquierda } \\
\text { (HVI) }\end{array}$ & $\begin{array}{l}\text { Enfermedad de Danon, PRKAG2, enfermedades } \\
\text { mitocondriales (MELAS) }\end{array}$ \\
\hline & PR corto/preexcitación & Enfermedad de Fabry tardía, amiloidosis \\
\hline & Bloqueo AV & Enfermedad de Danon, enfermedad de Pompe \\
\hline & Criterios de HVI extremo (Sokolov > $100 \mathrm{~ms}$ ) & Amiloidosis \\
\hline & Voltaje bajo de QRS & \\
\hline \multirow[t]{3}{*}{ MCD } & Bloqueo AV & Mutaciones lamina $\mathrm{A} / \mathrm{C}$, desmina, distrofia \\
\hline & Infarto posterolateral & miotónica, síndrome de Emery Dreifuss \\
\hline & & Distrofinopatías \\
\hline MCA & Ondas épsilon inferolateral & MCA con afectación biventricular \\
\hline & Onda $\mathrm{T}$ negativa inferolateral & \\
\hline MCR & Bloqueo AV & Desminopatías, amiloidosis \\
\hline
\end{tabular}

HVI: hipertrofia ventricular izquierda; MCA: miocardiopatía arritmogénica; MCD: miocardiopatía dilatada; MCH: miocardiopatía hipertrófica; MCR: miocardiopatía restrictiva.

Cribado específico de enfermedades metabólicas

\section{Enfermedad de Fabry}

Se detecta entre un 1-3\% de los pacientes inicialmente diagnosticados de MCH. Es una enfermedad lisosomal causada por la deficiencia de la enzima alfa-galactosidasa A. La herencia está ligada a X, con afectación precoz y más grave de los hombres (en torno a los 30 años de edad). Ante la sospecha clínica, es necesaria la demostración de una actividad muy baja o indetectable de la enzima alfa-galactosidada en plasma o leucocitos. La prueba de la gota de sangre seca es un método sencillo y específico. El análisis genético confirma el diagnóstico y es imprescindible para determinar si una mujer es heterocigota, ya que la actividad enzimática en ellas puede ser normal o ligeramente reducida ${ }^{6}$.

\section{Enfermedad de Danon}

En este caso existe una mutación del gen que codifica la proteína-2 asociada a la membrana lisosomal (LAMP-2). Se transmite con herencia ligada a X. Afecta a hombres jóvenes y a mujeres unas décadas después, siendo la edad de presentación variable. La afectación cardíaca cursa en forma de hipertrofia miocárdica concéntrica severa o con formas atípicas que presentan evolución rápida a disfunción sistólica grave. La prueba genética proporciona el diagnóstico definitivo ${ }^{7}$. 
Ecocardiografía transtorácica

Determinados hallazgos ecocardiográficos pueden sugerir un tipo de miocardiopatía determinada. De forma resumida, se presentan en la tabla $3^{5}$.

Tabla 3. Patrones de ecocardiografía transtorácica que pueden sugerir una miocardiopatía concreta

\begin{tabular}{lll}
\hline Fenotipo & Hallazgo & Enfermedad \\
\hline \multirow{2}{*}{ MCH } & HVI concéntrica & \\
& HVI concéntrica severa & Enfermedad de Fabry, glucogenosis \\
MCD & Ventrículo izquierdo no compactado, acinesia & MCD genética \\
& posterolateral/discinesia & Miocardiopatía relacionada con distrofina \\
MCA & Coexistencia disfunción ventrículo izquierdo (VI) & MCA con afectación biventricular \\
MCR & Obliteración parcial de ápex de VI/VD & Hipereosinofilia
\end{tabular}

HVI: hipertrofia ventricular izquierda; MCA: miocardiopatía arritmogénica; MCD: miocardiopatía dilatada; MCH miocardiopatía hipertrófica; MCR: miocardiopatía restrictiva.

Resonancia magnética cardíaca

Permite realizar una medición de los grosores y volúmenes ventriculares con fiabilidad, y contribuye a sugerir el diagnóstico etiológico mediante la identificación de ciertos patrones característicos.

1. Enfermedad de Fabry: hipertrofia ventricular concéntrica y realce tardío con gadolinio (RTG) inferolateral.

2. Mutaciones de la distrofina: RTG subepicárdico parcheado.

3. Miocardiopatía arritmogénica: reemplazo fibroadiposo del miocardio.

Estudio genético

Constituye una herramienta clave en el diagnóstico de las miocardiopatías familiares, teniendo además utilidad a la hora de ofrecer consejo reproductivo, profesional y organizar el seguimiento de los familiares. En algunos casos, la mutación identificada tiene implicaciones pronósticas (algunas mutaciones en el gen TNNT2 se asocian a MCH de alto riesgo) ${ }^{8}$, pudiendo llevar incluso a la toma anticipada de ciertas decisiones terapéuticas (como considerar el implante de un desfibrilador de forma precoz en pacientes con MCD portadores de una mutación en la lámina $\mathrm{A} / \mathrm{C})^{9}$.

\section{Conflicto de intereses}

Los autores declaran no tener ningún conflicto de intereses.

\section{Responsabilidades éticas}

Protección de personas y animales. Los autores declaran que para esta investigación no se han realizado experimentos en seres humanos ni en animales.

Confidencialidad de los datos. Los autores declaran que en este artículo no aparecen datos de pacientes. 
Derecho a la privacidad y consentimiento informado. Los autores declaran que en este artículo no aparecen datos de pacientes.

\section{Bibliografía}

1. Elliot P, Andersson B, Arbustini E, Bilinska Z, Cecchi F, Charron P, et al. Classification of the cardiomyopathies: a position statement from the european society of cardiology working group on myocardial and pericardial diseases. Eur Heart J. 2008;29(2):270-6.

2. Report of the WHO/ISFC Task Force on the Definition and Classification of Cardiomyopathies. Br Heart J. 1980;44(6)672-3.

3. Arbustini E, Narula N, Dec GW, Reddy KS, Greenberg B, Kushwa $\square$ ha S, et al. The $\operatorname{MOGE}(\mathrm{S})$ classification for a phenotype-genotype nomenclature of cardiomyopathy [published correction appears In J Am Coll Cardiol. 2014;63:191-4]. J Am Coll Cardiol. 2013;62:2046-72.

4. Barriales-Villa R, Gimeno-Blanes JR, Zorio-Grima E, Ripoll-Vera T, EvangelistaMasip A, Moya-Mitjans A, et al. Protocolo de actuación en las cardiopatías familiares: síntesis de recomendaciones y algoritmos de actuación. Rev Esp Cardiol. 2016;69:300-9.

5. Rapezzi C, Arbustini E, Caforio AL, Charron P, Gimeno-Blanes J, Heliö T, et al. Diagnostic work-up in cardiomyopathies: bridging the gap between clinical phenotypes and final diagnosis. A position statement from the ESC Working Group on Myocardial and Pericardial Diseases. Eur Heart J. 2013;34:1448-58.

6. Sachdev B, Takenaka T, Teraguchi H, Tei C, Lee P, McKenna WJ, et al. Prevalence of Anderson-Fabry disease in male patients with late onset hypertrophic cardiomyopathy. Circulation. 2002;105:1407-11.

7. Arad M, Maron BJ, Gorham JM, Johnson WH Jr, Saul JP, Pérez-Atayde AR, et al. Glycogen storage diseases presenting as hypertrophic cardiomyopathy. N Engl J Med. 2005;35:362-72.

8. Priori SG, Blomström-Lundqvist C, Mazzanti A, Blom N, Borggrefe M, Camm J, et al. 2015 ESC Guidelines for the management of patients with ventricular arrhythmias and the prevention of sudden cardiac death. Europace. 2015;17(11):1601-87.

9. Charron P, Arad M, Arbustini E, Basso C, Bilinska Z, Elliott P, et al. Genetic counselling and testing in cardiomyopathies: a position statement of the European Society of Cardiology Working Group on Myocardial and Pericardial Diseases. Eur Heart J. 2010;31(22):2715-26. 